\title{
Synaptophysin and caspase-3 expression on lumbar segments of spinal cord after sensorimotor restriction during early postnatal period and treadmill training
}

\author{
Felipe Stigger ${ }^{1, *}$, Silvia Barbosa ${ }^{2}$, Marília Rossato Marques ${ }^{3}$, Ethiane Segabinazi ${ }^{3}$, Otávio Américo Augustin², Matilde Achaval ${ }^{4}$, \\ Simone Marcuzzo ${ }^{4}$ \\ 'Department of Physiotherapy, Federal University of Health Sciences of Porto Alegre, Porto Alegre, Brazil \\ 'Laboratory of Comparative Histophysiology, Department of Morphological Sciences, Federal University of Rio Grande do Sul, Porto Alegre, Brazil \\ ${ }^{3}$ Postgraduation Program of Neurosciences, Institute for Basic Health Sciences, Federal University of Rio Grande do Sul, Porto Alegre, Brazil \\ 4Postgraduation Program of Neurosciences, Department of Morphological Sciences, ICBS, Federal University of Rio Grande do Sul, Porto Alegre, Brazil
}

The purpose of the current study was to investigate whether locomotor stimulation training could have beneficial effects on spinal cord plasticity consequent to sensorimotor restriction (SR). Male Wistar rats were exposed to SR from postnatal day 2 (P2) to P28. Control and experimental rats underwent locomotor stimulation training in a treadmill from P31 to P52. The intensity of the synaptophysin and caspase-3 immunoreaction was determined on ventral horn of spinal cord. The synaptophysin immunoreactivity was lower in the ventral horn of sensorimotor restricted rats compared to controls animals and was accompanied by an in- creased caspase-3 immunoreactivity. Those alterations were reversed at the end of the training period. Our results suggest that immobility affects the normal developmental process that spinal cord undergoes in early postnatal life influencing both pro-apoptotic and synapse markers. Also, we demonstrated that this phenomenon was reversed by 3 weeks of treadmill training.

Keywords: Developmental disuse, Apoptosis, Spinal cord plasticity, Treadmill walking training

\section{INTRODUCTION}

Children restricted to bed/wheelchair or simple subjected to limited activity due to neurological disorders usually suffer from impairments in motor tasks. Cerebral palsy (CP), a heterogeneous clinical condition resulting from a lesion in developing central nervous system (CNS), is considered the most common cause of functional disability in childhood (Foran et al., 2005, Himmelmann et al., 2005). Although the primary lesion involved in the CP's brain pathology is static, it is proposed that motor performance could be declined with time, due difficulties to perform voluntary movement imposed by spasticity and impaired motor control (Kerr Graham and Selber, 2003).

There are several animal models, mainly focused in maternal infections or perinatal asphyxia and hypoxic-ischemic injuries, try- ing to reproduce the developmental and motor phenotype reminiscent of human $\mathrm{CP}$ condition. Although most models reported deficts on motor performance, they were, however, not similar of those observed in human and, with time, pups were able to compensate functional limitations (Boksa et al., 1995; Poggi et al., 2005; Strata et al., 2004). A motor phenotype close to those described in $\mathrm{CP}$ patients were more consistently replicated in rats by sensorimotor restriction (SR) during the early postnatal stage (Coq et al., 2008; Stigger et al., 2011b). Those studies suggested that voluntary movement is crucial to the development of posture and locomotion during the maturation of the central and peripheral nervous system. Animal models of SR usually try to mimic the immobility imposed by spasticity and has been already shown to be a valuable model to understand underlying mechanisms of neural adaptations occurred in response to immobility. A series of
${ }^{*}$ Corresponding author: Felipe Stigger (DiD https://orcid.org/0000-0003-0901-310X Department of Physiotherapy, Federal University of Health Sciences of Porto Alegre, Rua Sarmento Leite, 245, Porto Alegre, Rio Grande do Sul 90050-170, Brazil Tel: +55-51-99903021, Fax: +55-51-33038876, E-mail: stigger@ufcspa.edu.br Received: February 21, 2018 / Accepted: April 22, 2018
This is an Open Access article distributed under the terms of the Creative Commons Attribution Non-Commercial License (http://creativecommons.org/licenses/by-nc/4.0/) which permits unrestricted non-commercial use, distribution, and reproduction in any medium, provided the original work is properly cited. 
studies using the SR procedure showed that SR impact proprioceptive feedback from the hind limbs during development and affects cortical structural and functional organization (Coq et al., 2008; dos Santos et al., 2017; Marcuzzo et al., 2010; Strata et al., 2004). Although central effects of disuse are evident, the functional impairment in motor tasks could be resulted by the combination of both central and peripheral factors. In fact, it has been previously proposed that the locomotor abnormalities of CP may not be merely derived from cerebral dysfunction, but also partially due disruption of the spinal cord networks (de Louw et al., 2002).

It is well established that during early postnatal period the rat spinal cord undergoes a continuous maturation (Vinay et al., 2000). Actuality, this is a critical period for the development of postural responses in the hind limbs (Brocard et al., 1999). Through this period, voluntary movement and proprioceptive feedbacks seems essential to motoneuronal development (Inglis et al., 2000), posture and locomotion in the rat (Westerga and Gramsbergen, 1993). In fact, recent experiments showed that a period of SR inhibited the growth-related increase in soma size of motoneurons in the ventral horn of the spinal cord (Stigger et al., 2011a) and induced atypical refinement of the sensorimotor circuitry (Delcour et al., 2018).

Based in our recent finding indicating that there is a critical period of activity-dependent plasticity in the developing CNS and that morphological aspects of motoneurons could be changed by disuse, the aim of the present study is investigate whether diminished sensorimotor stimulation, induced by SR, affects spinal cord plasticity and development by analyzing the expression of synaptophysin, an intrinsic synaptic vesicle membrane protein related to the activity-dependent synapse formation, and caspase-3, an pro-apoptotic molecules involved in programed cell death (PCD) during development, in the ventral horn of lumbar segments of spinal cord.

Additionally, since treatment of CP patients usually starts soon after diagnosis in order to potentialize motor skills and muscle strength gains (Damiano, 2006) and that treadmill training have been successfully used to prevent motor and morphological alterations on neuromuscular system after SR (Stigger et al., 2011b), the effects of treadmill training will be also assessed aiming to obtain new insights into the clinical approach on pathological conditions comprising developmental disuse.

\section{MATERIALS AND METHODS}

All experiments and animal use have been carried out in accor- dance with the guidelines laid down by National Institutes of Health Guide for the Care and Use of Laboratory Animals (National Research Council, 2011) and followed the recommendations of the Brazilian Society for Neuroscience, Committee of the School of Veterinary Surgery, University of Buenos Aires and the International Brain Research Organization. Animals were housed in standard boxes, with controlled temperature and humidity, under a light/dark cycle of $12 \mathrm{hr}$, with food and water available ad libitum. Approval from the ethical committee of Federal University of Rio Grande do Sul (2006631) was obtained for procedures conducted in this research. All efforts were done to minimize animal suffering as well as to reduce the number of animals.

\section{Experimental animals}

After birth, 10 male Wistar pups were randomly divided into four experimental groups $(n=5)$ : (a) Control rats not exposed to any experimental procedure (CT); (b) rats subjected to transient hind limb immobilization from P2 to P28 for 16 hr/day (SR); (c) animals not exposed to SR procedure that underwent locomotor stimulation training consisted by walking on a treadmill, with low speed, for 3 weeks from P31 to P52 (TrCT); and (d) animals subjected to SR and exposed to locomotor stimulation training (TrSR). For details of SR and locomotor stimulation protocols see Marcuzzo et al. (2010).

\section{Immunoistochemical procedure}

After treadmill training animals were deeply anesthetized and transcardially perfused with saline solution followed by a solution containing $4 \%$ paraformaldehyde (Reagen, Colombo, Brazil) diluted in $0.1 \mathrm{M}$ phosphate buffer ( $\mathrm{PB} ; \mathrm{pH}, 7.4$ ) at room temperature. L4-5 segments of spinal cord were removed and postfixed in a solution containing $4 \%$ paraformaldehyde in $0.1 \mathrm{M} \mathrm{PB}$ for $4 \mathrm{hr}$ and then cryoprotected in a $15 \%$ and $30 \%$ sucrose solution (Synth, Diadema, Brazil) at $4{ }^{\circ} \mathrm{C}$ until they sank. After cryoprotection segments were quickly frozen in isopentane (Merck, Darmstadt, Germany) cooled in liquid nitrogen and kept in a freezer $\left(-70^{\circ} \mathrm{C}\right)$ for further analyses. Transversal sections $(40 \mu \mathrm{m})$ were cut using a cryostat (Wetzlar, Leica, Germany) at $-20^{\circ} \mathrm{C}$ and collected in a PB saline (PBS), $\mathrm{pH}$ 7.4. The free-floating sections were washed in PBS, pretreated with 3\% hydrogen peroxide for $30 \mathrm{~min}$, washed again in PBS and after in PBS containing $0.4 \%$ Triton X-100 (PBS-Tx) for $15 \mathrm{~min}$, treated with 2\% bovine serum albumin (In lab, São Luís, Brazil) in PBS-Tx for 30 min an incubated with the primary antibody Monoclonal Anti-Synaptophysin or Caspase-3 (Sigma Chemical Co., St. Louis, MO, USA) diluted 1:200 in PBS- 
Tx for $48 \mathrm{hr}$ at $4^{\circ} \mathrm{C}$. The sections were again washed in PBS-Tx and incubated in the secondary antibody Anti-Mouse IgG-Peroxidase (Sigma Chemical Co.) diluted 1:500 for $2 \mathrm{hr}$ at room temperature. The reaction was revealed in a medium containing $0.06 \%$ 3,3-diaminobenzidine (DAB, Sigma Chemical Co.) dissolved in PBS for 10 min and after in $0.06 \%$ DAB with en $2 \mu \mathrm{L}$ of $3 \%$ hydrogen peroxide for $10 \mathrm{~min}$. The sections were washed in PBS, dehydrated in ethanol, cleared with xylene and covered with Entellan (Merck, Darmstadt, Germany) and coverslips. Control sections were prepared omitting the primary antibody by replacing it with PBS.

\section{Optical densitometry}

To measure the intensity of the synaptophysin and caspase-3 immunoreaction it was used a semiquantitative densitometric analysis. Digitalized images of the ventral horn of the spinal cords were obtained and converted to an 8-bit gray scale (0-255 gray levels) for further analysis. Picture elements (pixels) employed to measure optical density were obtained from tree areas of interest (AOI) measuring $3,775 \mu \mathrm{m}^{2}$ each overlaid on the gray scale image. All of the lighting conditions and magnifications were held constant. Both the left and right dorsal horn of spinal cord were used. For each animal, 30 measures were taken and the results shown were the total mean value from the three studied AOIs. Background staining subtraction and correction were done in accordance with our previous published protocol to calculate the optical density (Xavier et al., 2005).

\section{Neonatal developmental tests}

Briefly, after birth, daily evaluation of neonatal developmental milestones was by performed a blinded observer. Neonatal developmental milestones consisted in: (a) surface righting, (b) negative geotaxis, (c) cliff aversion, (d) forelimb grasp, (e) hind limb placing, and (f) open field activity. Each developmental test response was considered positive based on its first appearance. All measurements were time-limited to a maximum of 30 -sec period. For details of evaluation protocol see Poggi et al. (2005).

\section{Statistical analysis}

The data for each neonatal developmental tests were analyzed using unpaired Mann-Whitney statistics. Synaptophysin and caspase-3 expressionresults were analyzed using two-way analysis of variance with restriction and treadmill training as the independent variables followed by post hoc Duncan test. Data were expressed as means \pm standard error of the mean. Probability values less than $5 \%$ were considered significant. Statistical analysis was performed using STATISTICA ver. 12.0 (StatSoft, Tusla, OK, USA).

\section{RESULTS}

\section{Optical densitometry}

As observed in Fig. 1, the optical densitometry analysis of the lumbar segment showed that the synaptophysin immunoreactivity (synaptophysin-ir) was lower in the ventral horn of from the SR group $(0.182 \pm 0.002)$ when compared to either CT $(0.193 \pm 0.002)$,

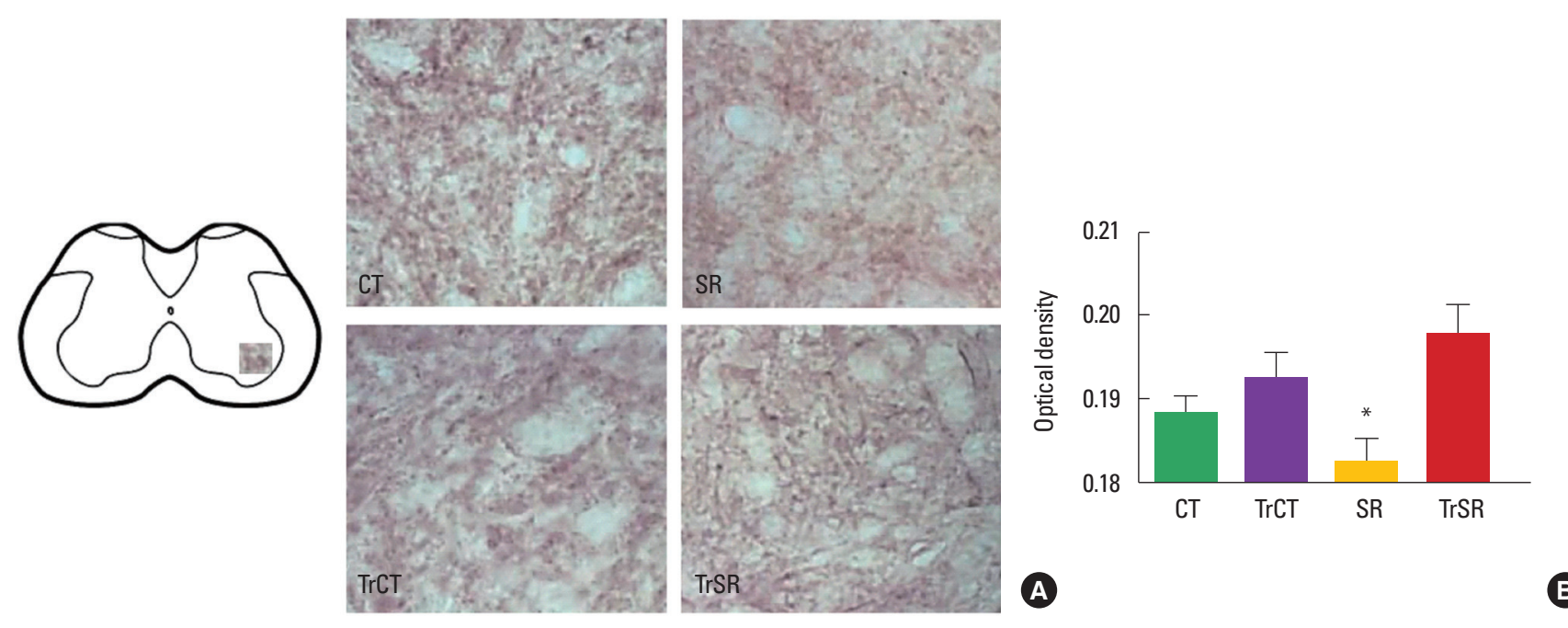

Fig. 1. Differential changes in synaptophysin immunoreactivity in the lumbar spinal cord of control (CT), sensorimotor-restricted (SR), trained control (TrCT), and trained sensorimotor-restricted (TrSR) rats. (A) Representative photomicrographs of of synaptophysin in ventral horn in all experimental groups ( $\times 20)$. (B) Two-way analysis of variance followed by Duncan test. Columns represent means \pm standard error of the mean. ${ }^{*} P<0.05$, different from CT. 

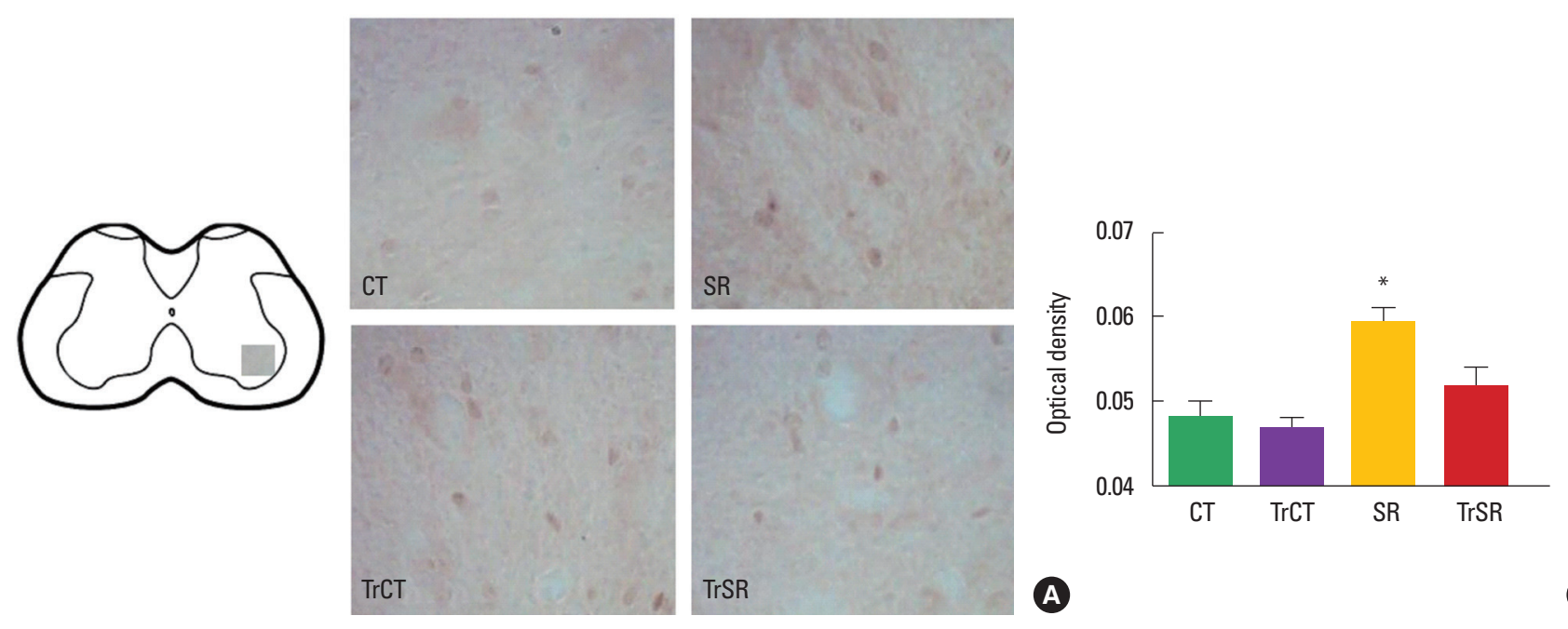

Fig. 2. Differential changes in caspase-3 immunoreactivity in the lumbar spinal cord of control (CT), sensorimotor-restricted (SR), trained control (TrCT), and trained sensorimotor-restricted (TrSR) rats. (A) Representative photomicrographs of of caspase-3 in ventral horn in all experimental groups $(\times 20)$. (B) Two-way analysis of variance followed by Duncan test. Columns represent means \pm standard error of the mean. ${ }^{*} P<0.05$, different from CT.

Table 1. Day of first performance of neonatal developmental sensory-motor behaviors in control (CT), sensorimotor-restricted (SR) animals

\begin{tabular}{lcc}
\hline Behavior & CT $(n=7)$ & SR $(n=8)$ \\
\hline Surface righting & $3.00 \pm 0.00$ & $3.00 \pm 0.00$ \\
Cliff aversion $^{\dagger}$ & $4.71 \pm 0.70$ & $9.25 \pm 0.67^{\dagger}$ \\
Negative geotaxis $^{\dagger}$ & $7.57 \pm 0.48$ & $10.25 \pm 0.52^{\dagger}$ \\
Hind limb placing $^{\dagger}$ & $4.42 \pm 0.61$ & $8.25 \pm 0.75^{\dagger}$ \\
Forelimb grasp $^{\text {Activity* }}$ & $3.00 \pm 0.00$ & $3.25 \pm 0.25$ \\
\hline
\end{tabular}

Values are presented as mean \pm standard error of the mean.

${ }^{*} P<0.05$, significantly different from $C T .{ }^{\dagger} P \leq 0.01$, significantly different from $\mathrm{CT}$.

CTTr $(0.192 \pm 0.002)$, or SRTr groups $(0.197 \pm 0.003)(P<0.05)$. There were no differences between the CT and CTTr groups, or between the CT and SRTr groups. Interestingly, the decreased synaptophysin-ir observed in the SR group was accompanied with a increased caspase-3 immunoreactivity (caspase3-ir). Caspase3-ir was increased within the ventral horn of SR animals $(0.181 \pm$ $0.001)$ when compared to CT $(0.170 \pm 0.001)$, CTTr $(0.169 \pm$ $0.001)$, or SRTr groups $(0.174 \pm 0.001)(P<0.05)$. Again, no differences were observed between the CT and CTTr groups, or between the CT and SRTr groups (Fig. 2).

\section{Neonatal developmental tests}

As showed in Table 1, sensorimotor restricted pups performed cliff aversion $(P<0.01)$, negative geotaxis $(P<0.01)$, hind limb placement $(P<0.01)$ and motor activity $(P=0.05)$ latter than CT animals. No differences were observed on surface righting and fore- limb grasp.

\section{DISCUSSION}

The present study provides evidence for a change in the maturation and plasticity of lumbar spinal cord in a activity-dependent manner following a 26-day period of SR and locomotor stimulation in rats at early stages of development. As results, first we found that the synaptophysin expression in the ventral horn of the L4-5 lumbar segments of spinal cord was lower in SR animals comparing to all experimental groups, this results were accompanied with an increased expression of caspase-3. Second, the locomotor stimulation program was able to reverse the alterations in synaptophysin and a lower caspase- 3 expression induced by SR. Also, SR induced changes in motor development. Taken together, these results suggest that motor activity is essential to the normal process of development, that includes synaptogenesis and PCD, and thus, these process could participate as substrates for the motor deficits observed in developmental disuse conditions such CP. Additionally, the present data encourage early locomotor stimulation in developmental disuse conditions considering that spinal cord plasticity alterations are reversible in response to treatment.

The rat spinal cord undergoes a substantial and continuous development in the course of pre/perinatal and early postnatal period. An initial phase of differentiation of motoneurons including fiber outgrowth and synapses formation initiates at the last week before birth (Altman and Bayer, 1984) and, following the next 
three weeks, motoneurons undergoes a rapid period of development. This period is accompanying by the establishment of mature and functional synapses of both afferent and efferent networks. Through this process, the storage and release of synaptophysin appears to be critical in regulating activity-dependent synapse formation (Tarsa and Goda, 2002) by stimulating proliferation, migration, fiber outgrowth and the establishment of transient and/or permanent synapses (Bergmann et al., 1991).

One of our results is the reduced expression of synaptophysin within the ventral horn of spinal cord in response to immobilization. In the rats' spinal cord, synaptophysin expression has been shown to start at embryonic day (ED) 12, reaching a continuous level, which is kept until birth, after ED 14. It's expression is followed by a postnatal increase reaching the adult pattern (Bergmann et al., 1991). In present study we demonstrate that the synaptophysin expression could be modulated for either decreased or increased motor activity. Synaptophysin expression seems to occur in an activity-dependent manner modulated by neurotrophic factors such as brain-derived neurotrophic factor (BDNF) and neurotrophin-3 (NT-3). At the time of nerve-muscle contact BDNF and NT-3, are expressed by embryonic muscle cells (Schecterson and Bothwell, 1992) and can contribute to establishment and maturation of neuronal synapses (Wang et al., 2010).

Not only, BDNF and NT-3 expression could be involved in changes on spinal circuitry. Further than peripheral stimulus, descending inputs that occur during practice also seems to be decisive in development of muscle afferents (Wolpaw and Tennissen, 2001). Following developmental disuse, remodeling within the somatosensory cortex (Coq et al., 2008) and motor cortex (Strata et al., 2004) occurs. Remodeling within central areas contribute to atypical projections to spinal motoneurons and consequently impair motoneuron development (Stigger et al., 2011a). Corticospinal inputs to motoneurons are essential in guiding the development of spinal cord circuitry by refining afferent connectivity and modulating connection between spinal interneurons (Clowry, 2007). Thus, changes in synaptophysin expression could be due peripheral mechanisms, but, also have supraspinal contributions since motor areas could be affected by disuse.

Our results also demonstrated that the SR-induced decrease in synaptophysin expression was accompanied to a delay in achieving some developmental milestones and locomotion. Synaptogenesis in rats' spinal cord seems to be strictly related with the acquisition of developmental milestones such locomotion and posture (Kerai et al., 1995). The rats' hind limbs are not motile at birth (Geisler et al., 1993). This, at least in some rodents, coincide to a period that synapses are not totally established in the gray matter of spinal cord (Gingras and Cabana, 1998). Actually, the synaptogenesis of spinal motoneurons with axons originated within the brain occurs mostly postnatally (Eyre, 2007) and only at the end of the first week pups become able to lift their trunk from the surface and to walk (Geisler et al., 1993). In rats, CNS axons arrive the spinal cord near postnatal day 0 (P0), reaching the lower cervical segments by P3 extending to all segments around the end of the second postnatal week (Stanfield, 1992). Consequently, the disturbance in activity level within early postnatal period due SR procedure could be responsible to alter normal development and acquisition of a mature pattern of motor behavior.

Besides synaptogenesis, programmed cell death (PCD) during usual development has been also described in both central and peripheral nervous systems resulting in the elimination of the initial number of cells (Oppenheim, 1991). In fact, during development, apoptosis in both embryonic and postnatal motoneurons was reported (Lowrie and Lawson, 2000). Cysteine proteases, including the caspase family, are pro-apoptotic molecules that induces PCD throughout development (Oppenheim et al., 2001). Our study demonstrated that SR increases the expression of caspase- 3 compared to on normal developmental rats within the ventral horn of the lumbar spinal cord, what could be related to motoneurons and/or interneurons death. Following nerve injury, death of developmental neurons within spinal cord seem to occur by apoptosis (Lawson and Lowrie, 1998). To our knowledge there are only few trials examining spinal cord apoptosis following non traumatic disuse such as limb immobilization or hind limb unloading. Although Islamov et al. (2011) did not report any sign of apoptosis within hind limb motoneurons during 35-day hind limb suspension, their results indicated the increased expression of antiapoptotic factors. It seems that this resistance of motoneurons to a decreased motor activity is not found in neonatal rats since we found an overexpression of caspase- 3 in motoneurons within ventral horn. In fact, previous studies from our laboratory showed that SR during development is able to alter motoneurons maturation (Stigger et al., 2011a) inducing reduction in mean soma size. In the same way that occurred to synaptophysin expression, both central and peripheral diminished/abnormal inputs could be related to changes in caspase- 3 expression. One point that should be noted is that, although this results could possibly be relate to amplified cell death, it seems that it is not permanent since locomotor stimulus could normalize this parameter.

Since neurotropic factors are involved not only in maturation and plasticity of the central and peripheral nervous system but 
also in neuronal survival (Constandil et al., 2011), a neurotrophic hypothesis could explain both the increased caspase- 3 and decreased synaptophysin expression during SR procedure and also explain the normalization of its expression after the training. Neurotrophic factors expression occur in a activity dependent manner resulting from neural activity (Neeper et al., 1996). The reduction of synaptophysin expression was reversed after the SR rats participated in treadmill exercise. Therefore, although the locomotor stimulation used in our study is considered to be a low intensity exercise, the demand that these animals were exposed during our training protocol was sufficient to normalize their activity to levels comparable to those of $\mathrm{CT}$ rats. Exercise affects the expression of several neurotrophic factors (Heo et al., 2001; Ilha et al., 2011). Also, BDNF has the ability to inhibit caspase- 3 activation and subsequent apoptosis protecting against neuronal injury (Han et al., 2000). Adictionally, insulin-like growth factor-I, that has been previously revealed decreased in spinal cord after immobilization (Suliman et al., 2001), has been found to exert neuroprotective functions, reducing PCD of motoneurons during development ( $\mathrm{Li}$ et al., 1994; Neff et al., 1993).

To conclude, our results demonstrates that immobility disturbs the normal developmental process that spinal cord undergoes in early postnatal life. Our data extend these results and demonstrate that locomotor stimulation may also take advantage in the developmental process related to spinal cord. Considering our results here and in our previous study, an altered plasticity in spinal cord may contribute to the functional impairments of rats exposed to developmental disuse. A better knowledge of spinal cord plasticity occurred during pathological process, such those observed in children with CP and other developmental conditions that induces inactivity, will permit appropriate rehabilitation strategies aiming to promote functional gains and also to prevent disability to be settled.

\section{CONFLICT OF INTEREST}

No potential conflict of interest relevant to this article was reported.

\section{ACKNOWLEDGMENTS}

We are very grateful to $\mathrm{PhD}$ Matilde Achaval, founder of the Laboratório Histofisiologia Comparada, for the laboratory's structure. This work was supported by the Brazilian funding agencies: CNPq, CAPES and FAPERGS (002097-2552/13-6-1).

\section{REFERENCES}

Altman J, Bayer SA. The development of the rat spinal cord. Adv Anat Embryol Cell Biol 1984;85:1-164.

Bergmann M, Lahr G, Mayerhofer A, Gratzl M. Expression of synaptophysin during the prenatal development of the rat spinal cord: correlation with basic differentiation processes of neurons. Neuroscience 1991; 42:569-582.

Boksa P, Krishnamurthy A, Brooks W. Effects of a period of asphyxia during birth on spatial learning in the rat. Pediatr Res 1995;37(4 Pt 1): 489-496.

Brocard F, Vinay L, Clarac F. Development of hindlimb postural control during the first postnatal week in the rat. Brain Res Dev Brain Res 1999; 117:81-89.

Clowry GJ. The dependence of spinal cord development on corticospinal input and its significance in understanding and treating spastic cerebral palsy. Neurosci Biobehav Rev 2007;31:1114-1124.

Constandil L, Aguilera R, Goich M, Hernández A, Alvarez P, Infante C, Pelissier T. Involvement of spinal cord BDNF in the generation and maintenance of chronic neuropathic pain in rats. Brain Res Bull 2011; 86:454-459.

Coq JO, Strata F, Russier M, Safadi FF, Merzenich MM, Byl NN, Barbe MF. Impact of neonatal asphyxia and hind limb immobilization on musculoskeletal tissues and S1 map organization: implications for cerebral palsy. Exp Neurol 2008;210:95-108.

Damiano DL. Activity, activity, activity: rethinking our physical therapy approach to cerebral palsy. Phys Ther 2006;86:1534-1540.

de Louw AJ, de Vente J, Steinbusch HP, Gavilanes AW, Steinbusch HW, Blanco CE, Troost J, Vles JS. Apoptosis in the rat spinal cord during postnatal development; the effect of perinatal asphyxia on programmed cell death. Neuroscience 2002;112:751-758.

Delcour M, Massicotte VS, Russier M, Bras H, Peyronnet J, Canu MH, Cayetanot F, Barbe MF, Coq JO. Early movement restriction leads to enduring disorders in muscle and locomotion. Brain Pathol 2018 Feb 13 [Epub]. https://doi.org/10.1111/bpa.12594.

Dos Santos AS, de Almeida W, Popik B, Sbardelotto BM, Torrejais MM, de Souza MA, Centenaro LA. Characterization of a cerebral palsy-like model in rats: analysis of gait pattern and of brain and spinal cord motor areas. Int J Dev Neurosci 2017;60:48-55.

Eyre JA. Corticospinal tract development and its plasticity after perinatal injury. Neurosci Biobehav Rev 2007;31:1136-1149.

Foran JR, Steinman S, Barash I, Chambers HG, Lieber RL. Structural and mechanical alterations in spastic skeletal muscle. Dev Med Child Neurol 2005;47:713-717.

Geisler HC, Westerga J, Gramsbergen A. Development of posture in the 
rat. Acta Neurobiol Exp (Wars) 1993;53:517-523.

Gingras J, Cabana T. The development of synaptophysin-like immunoreactivity in the lumbosacral enlargement of the spinal cord of the opossum Monodelphis domestica. Brain Res Dev Brain Res 1998;106:211215.

Kerr Graham H, Selber P. Musculoskeletal aspects of cerebral palsy. J Bone Joint Surg Br 2003;85:157-166.

Han BH, D'Costa A, Back SA, Parsadanian M, Patel S, Shah AR, Gidday JM, Srinivasan A, Deshmukh M, Holtzman DM. BDNF blocks caspase-3 activation in neonatal hypoxia-ischemia. Neurobiol Dis 2000;7:38-53.

Heo YR, Kang CW, Cha YS. Influence of exercise-training on insulin-like growth factor I and II in rats. Nutr Res 2001;21:1191-1199.

Himmelmann K, Hagberg G, Beckung E, Hagberg B, Uvebrant P. The changing panorama of cerebral palsy in Sweden. IX. Prevalence and origin in the birth-year period 1995-1998. Acta Paediatr 2005;94:287294.

Ilha J, Centenaro LA, Broetto Cunha N, de Souza DF, Jaeger M, do Nascimento PS, Kolling J, Ben J, Marcuzzo S, Wyse AT, Gottfried C, Achaval $M$. The beneficial effects of treadmill step training on activity-dependent synaptic and cellular plasticity markers after complete spinal cord injury. Neurochem Res 2011;36:1046-1055.

Inglis FM, Zuckerman KE, Kalb RG. Experience-dependent development of spinal motor neurons. Neuron 2000;26:299-305.

Islamov RR, Mishagina EA, Tyapkina OV, Shajmardanova GF, Eremeev AA, Kozlovskaya IB, Nikolskij EE, Grigorjev AI. Mechanisms of spinal motoneurons survival in rats under simulated hypogravity on earth. Acta Astronaut 2011;68:1469-1477.

Kerai B, Greensmith L, Vrbová G, Navarrete R. Effect of transient neonatal muscle paralysis on the growth of soleus motoneurones in the rat. Brain Res Dev Brain Res 1995;85:89-95.

Lawson SJ, Lowrie MB. The role of apoptosis and excitotoxicity in the death of spinal motoneurons and interneurons after neonatal nerve injury. Neuroscience 1998;87:337-348.

Li L, Oppenheim RW, Lei M, Houenou LJ. Neurotrophic agents prevent motoneuron death following sciatic nerve section in the neonatal mouse. J Neurobiol 1994;25:759-766.

Lowrie MB, Lawson SJ. Cell death of spinal interneurones. Prog Neurobiol 2000;61:543-555.

Marcuzzo S, Dutra MF, Stigger F, do Nascimento PS, Ilha J, Kalil-Gaspar PI, Achaval M. Different effects of anoxia and hind-limb immobilization on sensorimotor development and cell numbers in the somatosensory cortex in rats. Brain Dev 2010;32:323-331.

National Research Council, Division on Earth and Life Studies, Institute for Laboratory Animal Research, Committee for the Update of the Guide for the Care and Use of Laboratory Animals. Guide for the Care and Use of Laboratory Animals. 8th ed. Washington (DC): National Academies Press (US); 2011.

Neeper SA, Gómez-Pinilla F, Choi J, Cotman CW. Physical activity increases mRNA for brain-derived neurotrophic factor and nerve growth factor in rat brain. Brain Res 1996;726:49-56.

Neff NT, Prevette D, Houenou LJ, Lewis ME, Glicksman MA, Yin QW, Oppenheim RW. Insulin-like growth factors: putative muscle-derived trophic agents that promote motoneuron survival. J Neurobiol 1993; 24:1578-1588.

Oppenheim RW. Cell death during development of the nervous system. Annu Rev Neurosci 1991;14:453-501.

Oppenheim RW, Flavell RA, Vinsant S, Prevette D, Kuan CY, Rakic P. Programmed cell death of developing mammalian neurons after genetic deletion of caspases. J Neurosci 2001;21:4752-4760.

Poggi SH, Park J, Toso L, Abebe D, Roberson R, Woodard JE, Spong CY. No phenotype associated with established lipopolysaccharide model for cerebral palsy. Am J Obstet Gynecol 2005;192:727-733.

Schecterson LC, Bothwell M. Novel roles for neurotrophins are suggested by BDNF and NT-3 mRNA expression in developing neurons. Neuron 1992;9:449-463.

Stanfield BB. The development of the corticospinal projection. Prog Neurobiol 1992;38:169-202.

Stigger F, do Nascimento PS, Dutra MF, Couto GK, Ilha J, Achaval M, Marcuzzo S. Treadmill training induces plasticity in spinal motoneurons and sciatic nerve after sensorimotor restriction during early postnatal period: new insights into the clinical approach for children with cerebral palsy. Int J Dev Neurosci 2011a;29:833-838.

Stigger F, Felizzola AL, Kronbauer GA, Couto GK, Achaval M, Marcuzzo S. Effects of fetal exposure to lipopolysaccharide, perinatal anoxia and sensorimotor restriction on motor skills and musculoskeletal tissue: implications for an animal model of cerebral palsy. Exp Neurol 2011b; 228:183-191.

Strata F, Coq JO, Byl N, Merzenich MM. Effects of sensorimotor restriction and anoxia on gait and motor cortex organization: implications for a rodent model of cerebral palsy. Neuroscience 2004;129:141-156.

Suliman IA, Lindgren JU, Elhassan AM, Diab KM, Adem A. Effects of short- and long-term rat hind limb immobilization on spinal cord insulin-like growth factor-I and its receptor. Brain Res 2001;912:17-23.

Tarsa L, Goda Y. Synaptophysin regulates activity-dependent synapse formation in cultured hippocampal neurons. Proc Natl Acad Sci U S A 2002;99:1012-1016.

Vinay L, Brocard F, Pflieger JF, Simeoni-Alias J, Clarac F. Perinatal development of lumbar motoneurons and their inputs in the rat. Brain Res Bull 2000;53:635-647.

Wang Z, Liao D, Li C. Effects of brain-derived neurotrophic factor on syn- 
apsin expression in rat spinal cord anterior horn neurons cultured in vitro. Neural Regen Res 2010;5:1243-1248.

Westerga J, Gramsbergen A. Development of locomotion in the rat: the significance of early movements. Early Hum Dev 1993;34:89-100.

Wolpaw JR, Tennissen AM. Activity-dependent spinal cord plasticity in health and disease. Annu Rev Neurosci 2001;24:807-843.
Xavier LL, Viola GG, Ferraz AC, Da Cunha C, Deonizio JM, Netto CA, Achaval M. A simple and fast densitometric method for the analysis of tyrosine hydroxylase immunoreactivity in the substantia nigra pars compacta and in the ventral tegmental area. Brain Res Brain Res Protoc 2005;16:58-64. 